\title{
REDIBUJANDO EL MAPA ELECTORAL CHILENO: INCIDENCIA DE FACTORES SOCIOECONÓMICOS Y GÉNERO EN LAS URNAS
}

\author{
David Altman \\ Instituto de Ciencia Politica, Pontificia Universidad Católica de Chile
}

Resumen

Este artículo estudia el cambio de clivajes en el Chile post autoritario usando evidencia electoral y socioeconómica que va más allá de elecciones presidenciales y datos de opinión pública. Esta investigación determina que las condiciones socio económicas (medidas a través del Índice de Desarrollo Humano) tienen los efectos esperados en cuanto al porcentaje de votos recibidos por las dos coaliciones mayoritarias del país (la coalición gubernamental Concertación y el bloque opositor Alianza). En términos generales, la Concertación obtiene mejores resultados en las comunas de mayor índice de desarrollo humano y la Alianza en comunas con niveles más bajos de desarrollo humano. Si consideramos los efectos curvilíneos, la Alianza por Chile vota mejor en las comunas con valores extremos de IDH y la Concertación dentro de las comunas con valores medios. En otras palabras, la Concertación presenta una distribución con forma de $U$ invertida $(\cap)$ y la Alianza lo opuesto $(\cup)$. Respecto al voto de la mujer, se puede afirmar que, si bien tiende a favorecer a los partidos de la Alianza por Chile, simultáneamente tiende significativamente a votar más a las mujeres que a los hombres, dándose así una suerte de solidaridad de género.

Abstract

This article studies the change of cleavages in the post-authoritarian Chile using electoral and socioeconomic evidence that goes beyond presidential elections and public opinion polls. This research allows us to determine that the socioeconomic conditions (measured through the index of human development) have the expected effects on the percentage of votes obtained by each of the two major coalitions of the country (the governing Concertación, and the opposition Alianza). In general terms, the Concertación obtains better results in the communes with greater values of the index of human development and the Alianza in communes with lower levels of human development. Nonetheless, if we considered the curvilinear effects, the Alianza perform better in communes with extreme values of IDH and the Concertación in the communes with average values. In other words, the Concertación presents a distribution with a shape similar to an inversed $U(\cap)$, and the Alianza otherwise $(\cup)$. With respect to the vote of women we can affirm that, although they still tend to favor parties of the Alianza, women simultaneously tend to vote more to the women than to the men. Therefore, we can observe gender solidarity at the polls.

\section{PALABRAS CLAVE • Chile • Elecciones $\bullet$ Clivaje $\bullet$ Competencia • Género}

* $\quad$ Agradezco a la Dirección General de Postgrado, Investigación, Centros y Programa (DIPUC) de la Pontificia Universidad Católica de Chile, Proyecto 2003/07E2 y a la extraordinaria ayuda de José Manuel Morán. También quiero agradecer los comentarios y las sugerencias de Raul Elgueta, Miguel Angel López, Juan Pablo Luna, Aníbal Pérez-Liñán y Patricio Navia. 
En este trabajo se estudian tres puntos que la literatura ha explorado muy escuetamente en cuanto al voto de la ciudadanía en Chile usando los resultados de los comicios municipales del 2000 y de las elecciones legislativas del 2001. Este objetivo se planteará dando respuesta a tres preguntas: 1) ¿en qué medida los votos obtenidos por las dos grandes coaliciones dependen del impacto de variables socio económicas e institucionales?, 2) ¿en qué medida el voto femenino se inclina por alguno de los bloques políticos? y 3) ¿en qué medida se puede observar una solidaridad de género en las urnas?

Si bien Chile ha servido como un gran referente a nivel latinoamericano, poco se ha hecho en los últimos años sobre la base de datos electorales. Es claro que en el contexto latinoamericano Chile sobresale por tener un sistema de partidos altamente institucionalizado (Mainwaring y Scully, 1995; Siavelis, 2000), que quizás sea el más parecido de la región a sus contrapartes de la Europa continental en cuanto a su estructuración clasista (Coppedge, 1998; Dix, 1989). Por esta razón, dentro de la politología chilena una de las más prolíicas discusiones ha surgido en torno a cómo este sistema de partidos representa los clivajes sociales y su relevancia política contemporánea.

Muchos autores manifiestan que en Chile se puede seguir pensando como un sistema de tres tercios (izquierda-centro-derecha) (ver por ejemplo: Siavelis, 1997; Valenzuela, 1994; Valenzuela, 1995; Valenzuela, 1999), mientras que otros investigadores arguyen que existe una estructuración nueva y distinta de clivajes que surgió en torno a la experiencia militar (Agüero, Tironi, Valenzuela, y Sunkel, 1998; Carey, 2002; Mainwaring y Torcal, 2003; Ortega, 2003; Tironi y Agüero, 1999). Posiblemente, dependiendo de la pregunta de investigación, todos tengan parte de razón. Sin embargo, lo más interesante es que, a pesar de tener argumentos muy sólidos, pocos de estos estudios procuran hacer análisis electorales y cuando de evidencia empírica se trata, pocos van más allá del uso de encuestas de opinión pública o de resultados electorales presidenciales. Este artículo ofrece una nueva oportunidad de estudiar el cambio de clivajes en el Chile post autoritario usando evidencia electoral y socio económica que va más allá de elecciones presidenciales y datos de opinión pública.

La discusión de clivajes políticos se encuadra dentro de un tema más general que es el funcionamiento de la democracia chilena. Para algunos investigadores, como Nolte (2003), a pesar del escepticismo sobre la herencia del régimen militar y la combinación de presidencialismo y multipartidismo, el sistema político chileno no sólo parece estar funcionando mejor que sus vecinos del continente, sino que, como lo considera Angell (2003), ha mostrado un grado de estabilidad remarcable desde la transición a la democracia en 1990. Otros autores, sin embargo, como Godoy (2003) y Siavelis (2000; 2002), aluden a las especiales circunstancias de la transición, que forzando a los actores políticos a actuar colectivamente, favorecieron a los presidentes de turno. Para los del primer grupo, con más de 14 años de funcionamiento institucional continuo, parece débil el argumento que el sistema ha funcionado correctamente sólo por causas excepcionales; para los otros, la evidencia es inconclusa y mantienen una cuota de dudas sobre cómo el sistema político chileno sería capaz de enfrentar momentos de crisis.

\section{SISTEMA ELECTORAL MUNICIPAL Y LEGISLATIVO EN CHILE}

Esta sección esboza los principales elementos de los fundamentos de los sistemas electorales municipales y legislativos de Chile. Respecto al sistema municipal, el Artículo 99 de la Constitución de la República establece que para el gobierno y la administración interior del Estado, el territorio 
de la República se divide en regiones y éstas en provincias, y que para los efectos de la administración local las provincias se dividen en comunas. Por su parte, el artículo 107 establece que la administración local de cada comuna o de las agrupaciones de comunas que determine la ley reside en una municipalidad, que estará constituida por el alcalde, que es su máxima autoridad, y por el concejo. En la actualidad existen 346 comunas a lo largo de Chile, 345 con municipios, ya que la Antártica elige a sus concejales dentro de la municipalidad de Cabo de Hornos. Si bien existen enormes diferencias en magnitud poblacional entre las comunas, la comuna típica (promedio) tiene una población de 23.000 ciudadanos (oscila entre 306 y 173.593) y elige 6.21 concejales (por ley se eligen 6, 8 ó 10 concejales por comuna).

Según lo dispone el artículo 108 de la Constitución Política de Chile, las autoridades municipales que se elijan durarán cuatro años, pudiendo ser reeligidas. Para ser alcalde o concejal se requiere ser ciudadano con derecho a sufragio; saber leer y escribir; tener residencia en la región a que pertenezca la respectiva comuna a lo menos durante los últimos dos años anteriores a la elección; tener su situación militar al día, y no estar inhabilitado por la ley. A su vez, una persona no podrá postular simultáneamente a los cargos de alcalde o concejal, sea en la misma comuna o en comunas diversas (Art. 107, inciso $1^{\circ}$ Ley $\mathrm{N}^{\circ}$ 18.695) ${ }^{1}$.

Para las municipales del 2004 las reglas del juego cambiaron. En primera instancia, se desvincula la elección de alcaldes y de concejales (se vota en listas separadas y se permite lo que se denomina el voto cruzado). El otro gran cambio en comparación con las elecciones de 1992, 1996, y del 2000 es que la elección de alcaldes es por mayoría simple sin umbral mínimo y sin segunda vuelta. Los concejales son elegidos usando una formula de representación proporcional basada en la cifra repartidora de D'Hont. Si bien se utiliza un mecanismo de representación proporcional, las diferencias inter comunales en cantidad de electores impactan enormemente sobre la cantidad de votos necesarios para elegir un concejal. Así por ejemplo, el coste en votos más alto en votos es la Comuna de Maipú, Región Metropolitana, donde es necesario conseguir unos 17.936 votos, siendo la más "barata" la Comuna de Tortel (Región XI), donde con 51 votos se podría teóricamente conseguir una plaza en el concejo, siendo solamente 351 veces más barato en Tortel que en Maipú.

Las elecciones municipales ofrecen datos curiosos. Por ejemplo, en la elección del 2000, Jorge Canto Núñez de la UDI fue elegido concejal por la Comuna de Primavera en la Región XII con un voto, que representó el $0.13 \%$ del voto comunal. Otras ocho personas fueron elegidos concejales con un único voto. Lo más interesante es que hubo situaciones donde el porcentaje de votación fue aún menor que el del concejal Canto: el independiente Mauricio Camus Valverde fue elegido concejal por la Comuna de Las Condes con 97 votos, que representaron el 0,09\% del voto en su comuna. Contrariamente, el concejal que "pagó" más cara su plaza fue Carlos Alarcón Castro de Peñalolén, que con 44.786 votos se convirtió en alcalde. El problema de todos estos datos anecdóticos es que aún carecemos de teorías para explicar por qué en algunos casos estos

Como dice Navia: "Para resultar elegido alcalde en 1996 y 2000, se necesitaba la mayor votación personal entre todos los candidatos a concejales y pertenecer a una lista que haya obtenido más del $30 \%$ de los votos. De esta forma, en ocasiones el candidato ganador no pertenecía al partido/coalición con más votos. Los incentivos de la ley electoral también convertían la elección de concejales en un trámite secundario a la elección de alcalde. Importaba mucho más ganar un alcalde que obtener una mayoría de los concejales. Por eso mismo, los simpatizantes de una coalición tenían incentivos para concentrar sus votos en un solo candidato y así maximizar la posibilidad de asegurar la alcaldía" (Navia, 2003), ver también Navia (2002). 
individuos fueron elegidos con tan pocos votos e incluso carecemos de teorías que nos ayuden a entender por qué una coalición, por ejemplo, vota mucho mejor en un distrito que en otro.

Debido a la extensa literatura existente sobre el sistema electoral en el legislativo, menor énfasis haré sobre éste aquí. Sin embargo, brevemente, podemos señalar que en el llamado sistema binominal chileno los partidos políticos o coaliciones de partidos compiten en 60 distritos electorales compuestos por dos miembros cada uno. Así como los concejales, los diputados son elegidos usando una variante de la cifra repartidora de D'Hont. Los partidos y coaliciones usualmente presentan dos candidatos para cada distrito. Cada ciudadano vota por una única persona. El primer candidato elegido es aquel que recibió más votos del partido o coalición que recibió más votos. El segundo candidato elegido es del mismo partido del primero siempre y cuando su partido o coalición duplique o más que duplique al partido que lo sigue inmediatamente en número de votos. Usualmente se menciona que con un $33.4 \%$ del voto, el segundo partido o coalición logra hacerse del $50 \%$ de los representantes del distrito. Esto es cierto bajo la premisa que sólo dos partidos o coaliciones reciban votos en un distrito dado. En la gran mayoría de distritos chilenos compiten otros partidos (quizás el más significativo para ejemplificar sea el Partido Comunista), que logran un número no menor de votos. Por lo tanto, el pensar en $33.4 \%$ del voto en el distrito puede inducirnos a confusiones. Asumiendo que, en términos generales, los partidos o coaliciones minoritarios ganan aproximadamente un $10 \%$ del voto, el porcentaje necesario para que el segundo logre obtener la mitad de los candidatos en un distrito es apenas superior al 30\% del voto, generando tremendas distorsiones en la representatividad del sistema y ciertamente disminuyendo los incentivos a participar ya que el resultado, salvo raras excepciones, es fácil de anticipar².

\section{NIVEL SOCIOECONÓMICO EN LA DISTRIBUCIÓN DEL VOTO ENTRE LOS BLOQUES POLITICOS}

Las motivaciones detrás del voto han generado una enorme literatura y en su forma más simplista se pueden señalar dos grandes escuelas: la de identificación partidaria y la del voto económico ${ }^{3}$. De acuerdo con aquellos que suscriben los modelos de voto económico, las variables de índole económica afectarían significativamente el resultado de la votación (aunque hay que reconocer que la mayoría

2 A medida que el número de partidos o coaliciones en un distrito aumenta, el porcentaje necesario para lograr la segunda banca disminuye. Así por ejemplo en una configuración 49\%, 26, y 25, sólo los dos primeros partidos obtienen una banca cada uno. Más aún, el porcentaje de voto de los partidos o coaliciones fuera de los dos grandes pactos está significativamente correlacionado con la cantidad de votantes que existan en un distrito dado (Pearson R de 0.17 significativo al 0.01\%). Sobre el sistema binominal ver Siavelis (2004); Ortega (2003); Nolte (2003); Navia (2004); Angell (2003); Carey (1997); Carey (2003); Dow (1998); Dow (1998); Rahat (1998), Carey y Siavelis (2003).

3 Algunas perspectivas van mucho más allá de estos factores de identificación y económicos. A modo ejemplo, en Estados Unidos se ha estudiado que la participación local es altamente sensible a la distribución etaria y de género de una población. Según Gimpel, Morris y Armstrong, la participación es notablemente superior en el grupo mayor de 60 años, cayendo radicalmente en el grupo que oscila entre 18 y 29 años de edad (Gimpel, Irwin L. Morris, y Armstrong, 2004). Estudiando más de 4000 elecciones locales en Gran Bretaña entre 1983 y 1999 Rallings, Thrasher y Borisyuk (2003) llegan incluso a analizar el efecto climático sobre el voto local, mostrando que el invierno tiene un efecto negativo en la participación. Viniendo más a nuestro continente, Rowland (2001) estima el tamaño poblacional de las comunas de México y Bolivia como critico para determinar el éxito de los progresos en los programas de descentralización. Sin embargo para Garman, Haggard, and Willis (2001) es el grado de accountability que existe entre el gobierno descentralizado y el gobierno nacional el determinante de estos programas (ver tambien Blair, 2000). Estudios sobre la India indican a su vez que la democratización del gobierno local poco tiene que ver con el grado del capital social en las comunas, sino que con el grado de accountability que exista en las mismas (Sundar, 2001). 
sí toma en cuenta alguna variable política) ${ }^{4}$. Como evidencia de esta escuela se pueden subrayar las conocidas funciones voto-popularidad, a veces llamadas VP-functions, las cuales se han desarrollado en democracias europeas. En este ámbito encontramos los trabajos de Schneider, Pommerehene, Bruno and Frey (1981); Schneider y Neumann (1982), Nannestad y Paldman (1994), asi como también en los Estados Unidos Hibbs (1979), Eulau y Lewis-Beck (1985), Bowler y Donovan (1998), entre otros, y un pequeño grupo de investigaciones en América Latina (Altman, 2002; Araos y Engel, 1989; Panzer y Paredes, 1991; Rius, 1992). Dentro del ámbito de las instituciones, el trabajo de Jackman (1987) ha sido uno de los más fructíferos en lo que atañe a participación. Según Jackman (1987), el tamaño de los distritos electorales tiene un impacto claro sobre la participación: a mayor magnitud de distrito, mayor participación. Así también muestra cómo el multipartidismo tiene un efecto negativo en la participación. Más aún, a mayor desproporcionalidad en la asignación de escaños, menores son los índices de participación electoral (Jackman y Miller, 1995).

Es de suponer que la distribución del voto entre las dos grandes coaliciones se vea mediada por una variable de corte institucional. El propio sistema electoral aplicado para la elección de concejales y diputados incentiva la concentración del voto en una persona y ésta "gotea" a sus colegas de coalición aumentando el número de candidatos elegidos. En el ámbito municipal, por ejemplo, cuanto más concentrado el voto de una coalición esté en un único candidato, mayor será el porcentaje de votación que esta coalición recibe en la comuna en consideración. En otras palabras, la concentración de votos beneficia la performance electoral de los bloques políticos en el ámbito comunal. Para dar cuenta de este factor calcularé, usando la clásica formula de Laakso y Taagepera (1979), el número efectivo de candidatos de cada uno de los bloques a nivel municipal, hipotetizando que a menor número efectivo de candidatos por coalición, mejores serán los resultados electorales de esta coalición en ese determinado distrito o comuna ${ }^{5}$.

Por su parte, es de esperar que la participación electoral también afecte la distribución del voto inter coalicional. Una de las teorías más populares para explicar la participación electoral viene de la mano de lo que se denomina los modelos de "estatus socioeconómico" (SES) (Almond y Verba, 1963). Básicamente esta teoría de alcance medio indica que los individuos con más recursos económicos y sociales se interiorizan más en el ámbito de lo publico, participan más en los procesos electorales y, en consecuencia, son el núcleo "duro" de la política contemporánea (Lijphart, 1997; Powell, 1986). Contrariamente, los más débiles en recursos socioeconómicos presentan índices más altos de desafección. Si bien algunas opiniones esgrimen que la baja participación puede reflejar una alta satisfacción con el régimen político, la evidencia claramente sugiere que los individuos con menos oportunidades son aquellos que están menos inclinados a votar (Crewe,

4 Ha sido demostrado que, en general, el elemento económico dentro de la conducta electoral del ciudadano en las funciones voto-popularidad esta basado en un modelo sociotrópico. Esto es, que el ciudadano determina su voto por sus percepciones de la conducta macro económica más que egotrópico, o sea, en sus percepciones de sus propias y privadas condiciones materiales (Nannestad and Paldman, 1994: 224).

5 El índice de Laakso y Taagepera (1979) es el más ampliamente utilizado para medir la fragmentación de un sistema de partidos y este es extrapolable a otros escenarios para medir fragmentación.

$E N C=\frac{1}{\sum C_{i}^{2}}$

En este caso, ENC es el número efectivo de candidatos de una coalición dada y $C$ es el porcentaje de votos recibidos por cada candidato de esa coalición en una comuna o distrito determinado. La información electoral fue recabada del SERVEL, que es el órgano superior de la administración electoral en Chile (http://www.servel.cl) y del Sistema de Despliegue de Cómputos del Ministerio del Interior en (http://www.elecciones.gov.cl/). 
1982; Wolfinger y Rosenstone, 1980). Según Fornos, Power, y Garand (2004: 4) existen razones suficientes para que indicadores agregados de performance económica afecten la participación electoral ${ }^{6}$. En Chile es plausible esperar que aquellas comunas con mejores indicadores socioeconómicos tengan una participación electoral superior que las comunas con menor desarrollo socioeconómico, una variable tradicionalmente omitida a la hora de estudiar las diferencias en el porcentaje de ciudadanos en las urnas (ver Blais y Dobrzynska, 1998), y que a su vez este voto sea más de derecha (en otras palabras, que el voto sea más aliancista que concertacionista).

Para dar cuenta de la participación electoral usaremos el porcentaje de ciudadanos que acudieron a las urnas versus aquellos inscritos. Ello teniendo conciencia que éste no es el indicador por excelencia usado en la ciencia política comparada para medir la participación electoral sobre la población adulta en edad de votar. Lamentablemente, estos datos para Chile presentan falencias importantes por todo lo señalado en el apartado que detalla el problema que surge entre los datos de inscripción y los censos municipales.

La evidencia con que gozamos en Chile indica una tendencia clara en el sentido de que los niveles de desarrollo humano afectan directamente el tipo de voto. Mas aún, esta evidencia señala, en términos generales, un continuismo entre lo ocurrido antes del golpe de Estado en 1973 y el escenario post dictatorial. Según González Leiva: "Se aprecia en términos generales una fuerte vinculación entre la condición urbana de la población, volumen de la concentración, alfabetismo y los bajos niveles de población en extrema pobreza, con el apoyo que recibe la centro izquierda y la condición rural, la escasa concentración de población, el analfabetismo y los altos niveles de población en extrema pobreza con la centro derecha" (1999: 137) ${ }^{7}$. Por condiciones socioeconómicas en este trabajo usaremos como proxy el índice de desarrollo humano (IDH) elaborado por el PNUD de Chile en el año 2000 (ver Tabla 1) ${ }^{8}$.

TABLA 1: Índice de Desarrollo Humano Comunal en Chile (variables e indicadores)

\begin{tabular}{llll}
\hline Variable & Salud & Educación & Ingresos \\
\hline Indicador & $\bullet$ Años de vida & - Alfabetismo & - Promedio per cápita de los \\
& potencial perdidos & - Años de escolaridad & ingresos autónomos del hogar \\
& & promedio & - Desigualdad en la distribución \\
& & - Matriculación & del ingreso \\
& & combinada & - Incidencia de la pobreza de \\
& & ingresos \\
\hline
\end{tabular}

Fuente: PNUD Chile (2000: 9).

$6 \quad$ Si bien los modelos de participación han tradicionalmente enfatizado niveles agregados de desarrollo económico más que la performance en sí misma, Radcliff (1992) nos muestra que este tipo de variables -como cambio en el producto per cápita- afectan de forma disímil sobre índices de participación de los países desarrollados y los que no lo son. Según Radcliff, en los países en desarrollo, la performance económica está inversamente relacionada con la participación electoral, de forma tal que cuando las cosas están mal, los ciudadanos tienden a votar en gran medida (p. 445).

$7 \quad$ En Chile, uno de lo estudios "clásicos" que estudia el impacto de la mortalidad infantil, analfabetismo, sindicalización sobre los resultados electorales es el de Cruz-Coke (1958). Ya entrada esta nueva etapa post transición a la democracia, la mayor parte de los trabajos en esta avenida provienen de la geografía política. Ver también Aldunate (1981).

8 Los datos municipales por su lado provienen básicamente de dos fuentes: el SINIM, que es un sistema de información que sistematiza y reúne un conjunto de variables e indicadores en el tiempo, relativos al quehacer y gestión municipal (http://www.sinim.cl), y por otro lado se recogieron datos del Informe de Desarrollo Humano en las comunas de Chile del PNUD (2000), que abarca todas las comunas continentales del país (ver http://www.desarrollohumano.cl/otraspub/ Pub04/Desarrollofinal.pdf). 
Dicho esto, en los últimos años se ha evidenciado una muy alta penetración electoral del bloque de oposición en ámbitos que tradicionalmente pertenecían a la izquierda y que tienen niveles socioeconómicos bajos (Barozet, 2003; Luna, 2004). Si esto es así, la linearidad esbozada en el párrafo anterior se vería trastocada y en consecuencia es de esperar que el voto para candidatos de la coalición conservadora "Alianza" (derecha) presente una distribución con forma de "U" en función del índice de desarrollo humano. En otras palabras: el bloque conservador votaría mejor en ambos extremos del IDH y sería lógico pensar que tendríamos que observar una distribución casi inversa del voto para la coalición de gobierno (una $\mathrm{U}$ invertida). Ver Figura 1 por los efectos que la literatura esgrime (figura A) y los efectos esperados por esta investigación del IDH sobre el voto por bloques (figura B).

FIGURA 1: Relación esperada entre IDH y voto por coalición (Concertación y Alianza)
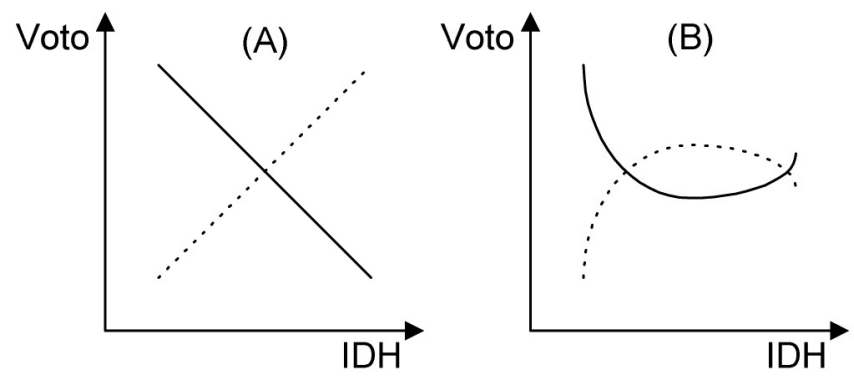

Asimismo, cabe esperar que el voto obtenido por las coaliciones esté mediado por el tipo de elección (municipales y legislativas), siendo el "efecto vecino" más importante que el ideológico-partidario. Debido a que esto es una hipótesis plausible, incluiremos en nuestro estudio una variable dicotómica (dummy) para controlar por este efecto. Además, en un país tan variado geográficamente como Chile, es de esperar que existan diferencias inter regionales. Por este motivo incluiremos una serie de variables dicotómicas para controlar por las diferentes regiones de la república.

Para testear las hipótesis convenidas haremos uso de regresiones multivariadas (OLS). Siguiendo los pasos de Mainwaring y Pérez-Liñán en su estudio sobre las determinantes de democracia en América Latina, aquí también uso la cuadrática de Indice de Desarrollo Humano como variable independiente para captar este efecto con forma de "U". Teóricamente asumo que la derecha vota mejor en municipios donde el IDH es bajo, pero además quiero captar el efecto que vota muy bien en las puntas del continuo (una U). La Concertación tendría una distribución contraria (vota muy bien en municipalidades "medias"). La variable dependiente en cada uno de los modelos es el porcentaje del voto recibido por cada una de las coaliciones en cada una de la totalidad de las unidades geográficas estudiadas por elección: para las elecciones municipales se toma el municipio y para las elecciones legislativas el distrito electoral. Ver Tabla 2. 
TABLA 2: Causas institucionales y socioeconómicas del porcentaje de voto recibido por las coaliciones en Chile (coeficientes no estandarizados y errores estándar)

\begin{tabular}{|c|c|c|c|c|}
\hline & $\begin{array}{c}\text { Modelo } 1 \\
\text { CONCERTACION }\end{array}$ & & $\begin{array}{c}\text { Modelo } 2 \\
\text { ALIANZA }\end{array}$ & \\
\hline (Constante) & $-1.178^{*}$ & .499 & $2.953^{* * *}$ & .467 \\
\hline $\mathrm{IDH}$ & $5.178^{* * *}$ & 1.365 & $-7.101^{* * *}$ & 1.275 \\
\hline $\mathrm{IDH}^{2}$ & $-4.004^{* * *}$ & .939 & $5.387^{* * *}$ & .877 \\
\hline Participación & -.023 & .050 & .073 & .047 \\
\hline \% Mujeres & .345 & .192 & $-.388^{*}$ & .179 \\
\hline Voto Total & $2.788 \mathrm{E}-07$ & .000 & $-2.923 E-07$ & .000 \\
\hline Legislativas & $-.080^{* * *}$ & .013 & $.026^{* *}$ & .011 \\
\hline | Región & -.013 & .036 & -.004 & .034 \\
\hline || Región & $.107^{* * *}$ & .035 & $-.081^{* *}$ & .033 \\
\hline |ll Región & -.007 & .021 & $.058^{* \star *}$ & .019 \\
\hline IV Región & .016 & .028 & -.020 & .026 \\
\hline V Región & -.025 & .021 & $.055^{\star *}$ & .020 \\
\hline VI Región & .035 & .022 & .001 & .021 \\
\hline VII Región & -.015 & .024 & $.068^{* * *}$ & .022 \\
\hline VIII Región & .040 & .022 & .009 & .020 \\
\hline IX Región & -.014 & .025 & $.097^{* * *}$ & .023 \\
\hline XI Región & -.065 & .038 & $.075^{\star}$ & .035 \\
\hline XII Región & $.118^{*}$ & .049 & -.070 & .046 \\
\hline Núm. Efec. de Cand. Concertación & $-.045^{* * *}$ & .008 & - & \\
\hline Núm. Efec. de Cand. Alianza por Chile & - & & $-.063^{* * *}$ & .007 \\
\hline $\mathrm{R}^{2}$ & .173 & & .291 & \\
\hline $\mathrm{R}^{2}$ Ajustado & .150 & & .271 & \\
\hline $\mathrm{F}$ & $7.477^{* * *}$ & & $14.714^{* * *}$ & \\
\hline $\mathrm{N}$ & 663 & & 663 & \\
\hline
\end{tabular}

${ }^{*} \mathrm{p}<.05 ;{ }^{* *} \mathrm{p}<.01 ;{ }^{* * *} \mathrm{p}<.005$

Los resultados satisfacen holgadamente las hipótesis planteadas. El modelo 1, que estudia el porcentaje de los votos recibidos por la Concertación en las elecciones del 2000 y del 2001, evidencia claramente la relación de $U$ invertida (dada por el coeficiente negativo de $\mathrm{IDH}^{2}$ ). Esto no significa que el IDH lineal no tenga impacto, todo lo contrario. El coeficiente positivo del IDH nos esta indicando que, ceteris paribus, la Concertación obtiene mejores resultados en las comunas de mayor índice de desarrollo humano. Evidentemente no podemos dejar de observar que los coeficientes de estas variables para el porcentaje de votos obtenidos por la Alianza son no solo altamente significativos, sino que además poseen los signos invertidos. En otras palabras, la Alianza vota mejor en términos generales en las comunas con valores extremos de IDH, pero también tiende a votar mejor en comunas con bajos niveles de desarrollo humano. De esta forma, se pueden avalar los efectos lineales de los estudios realizados por González Leiva (1999). 
Asimismo, la relación inversa entre los coeficientes (significativos ambos) del tipo de elección en consideración indica que en términos generales la Concertación vota mejor en elecciones locales (municipales) y la Alianza encuentra mejores resultados en las legislativas. Ambos modelos también dan cuenta de la importancia de la concentración de votos en pocos candidatos. Cuanto menor es el número efectivo de candidatos compitiendo en los municipios, mayores son los porcentajes que obtienen ambas coaliciones. Si bien en el ámbito regional es más complicado hacer conjeturas, es evidente que cuando el coeficiente de la región en cuestión es en ambos modelos significativo, éstos tienen pendientes opuestas (el único caso en la ll Región).

Sin embargo, es de suponer que, como dice Navia (comunicación personal), se pierde algo cuando comparamos a Puente Alto o Maipú (500 mil habitantes) con Cabo de Hornos, por ejemplo. Las comunas chilenas varían mucho en tamaño y esto puede estar influenciando enormemente los resultados de las regresiones presentadas. Para esquivar este problema existirían dos caminos alternativos. El primero sería ponderar por la cantidad de población de cada comuna, aunque esta solución crearía problemas estadísticos de otro tipo. Una segunda solución es simplemente considerar a las 100 comunas más pobladas del país, que concentran más de un $80 \%$ de la población del país. Este segundo camino es el adoptado en este trabajo. De esta forma estaríamos descartando las comunas que se encuentran en los extremos país, donde existe un conocido fenómeno migratorio más otras distorsiones (por ejemplo, son estas comunas donde muchos conscriptos residen, los inscriben y después no votan más). Si bien el universo de análisis se reduce, es de prever que los resultados de los modelos, eliminando el ruido producido por las distorsiones señaladas, mejore respecto a la realidad (ver Tabla 3). 
TABLA 3: Causas institucionales y socioeconómicas del porcentaje de voto recibido por las coaliciones en Chile en comunas de más de 30.000 inscritos (coeficientes no estandarizados y errores estándar)

\begin{tabular}{|c|c|c|c|c|}
\hline & $\begin{array}{c}\text { Modelo } 3 \\
\text { Concertación }\end{array}$ & & $\begin{array}{c}\text { Modelo } 4 \\
\text { Alianza }\end{array}$ & \\
\hline (Constante) & -1.237 & .889 & $2.951^{* * *}$ & .826 \\
\hline IDH & $4.970 *$ & 2.290 & $-6.814^{* * *}$ & 2.119 \\
\hline $\mathrm{IDH}^{2}$ & $-4.107^{* * *}$ & 1.470 & $5.367^{* * *}$ & 1.360 \\
\hline Participación & -.052 & .054 & .062 & .050 \\
\hline \% Mujeres & .990 & .606 & -.731 & .563 \\
\hline Voto Total & $3.495 \mathrm{E}-07$ & .000 & $-2.699 \mathrm{E}-07$ & .000 \\
\hline Legislativas & $-.093^{* * *}$ & .020 & .016 & .015 \\
\hline | Región & -.040 & .064 & -.086 & .059 \\
\hline || Región & .077 & .063 & -.017 & .059 \\
\hline ||| Región & -.009 & .030 & $.057^{*}$ & .028 \\
\hline IV Región & .048 & .039 & -.060 & .037 \\
\hline V Región & $-.056^{\star}$ & .029 & $.063^{* *}$ & .026 \\
\hline VI Región & -.036 & .037 & .014 & .034 \\
\hline VII Región & -.056 & .033 & $.076^{* *}$ & .031 \\
\hline VIII Región & .014 & .030 & .016 & .028 \\
\hline IX Región & .012 & .042 & $.077^{\star}$ & .039 \\
\hline XI Región & .077 & .104 & -.028 & .096 \\
\hline XII Región & $.201^{\star}$ & .094 & -.135 & .087 \\
\hline Núm. Efec. de Cand. Concertación & $-.061^{* * *}$ & .013 & - & - \\
\hline Núm. Efec. de Cand. Alianza por Chile & - & - & $-.066^{* *}$ & .011 \\
\hline $\mathrm{R}^{2}$ & .337 & & .450 & \\
\hline $\mathrm{R}^{2}$ Ajustado & .277 & & .401 & \\
\hline $\mathrm{F}$ & $5.629 * * *$ & & $9.054^{* * *}$ & \\
\hline $\mathrm{N}$ & 217 & & 217 & \\
\hline
\end{tabular}

${ }^{*} \mathrm{p}<.05 ;{ }^{* *} \mathrm{p}<.01 ;{ }^{* \star *} \mathrm{p}<.005$

Efectivamente, la linearidad de ambos modelos aumenta habiendo eliminado las comunas muy pequeñas del país (observado a través del aumento de $\mathrm{R}^{2}$ y el $\mathrm{R}^{2}$ Ajustado, comparando el Modelo 1 con el Modelo 3 y el 2 con el 4 respectivamente). Los coeficientes estudiados mantienen su significancia estadística, salvo en el Modelo 4, donde la variable que controla por tipo de elección (legislativa) pierde su significancia.

Probablemente a esta alturas surgirán preguntas relacionadas con el fenómeno partidario y no meramente coalicional; por ejemplo: en qué medida el índice de desarrollo humano afecta el voto a nivel de partido en el ámbito municipal. Aquí nos enfrentamos a un problema no menor sobre la unidad de análisis a considerar (partido frente a coalición). El actual sistema electoral imperante en Chile limita al investigador por la sencilla razón de que los resultados electorales medidos al 
nivel de partidos pueden estar confundiéndonos en cuanto a las lealtades partidarias de los electores. Es sabido que el sistema electoral tiene una enorme influencia en la distribución de candidatos en cada municipio y distrito (Siavelis, 2004). Así, por ejemplo, en una comuna o en un distrito los candidatos preferenciales de la CPD pueden ser del PPD y en la elección "E", y en el mismo distrito o comuna en la elección " $E+1$ " este candidato pertenezca al PDC9. No sería de extrañarse que un concertacionista miembro del PRSD vote por el PPD y el PDC en cada una de estas elecciones. En el primer caso estaríamos "inflando" al PPD y en el segundo caso al PDC, cuando en realidad este ciudadano mantiene su lealtad al PRSD disminuyendo su caudal de forma considerable en ambas elecciones. Es plausible que muchos concertacionistas leales voten por el candidato de turno de la CPD en este municipio, por más que ellos y ellas mantengan su lealtad con su partido. Si esto es correcto, es de esperar que si estudiamos el porcentaje de votos que recibe cada partido usando las mismas variables independientes que en los modelos anteriores, estas variables nos den una muy baja significancia estadística.

Otro cambio relevante a la hora de hacer comparaciones entre los modelos es la significancia del porcentaje de mujeres en cada una de las unidades geográficas entre el Modelo 2 y el Modelo 4. En este último modelo, esta variable pierde su significancia estadística, inhabilitándonos para hacer inferencias sobre su impacto en la votación por uno u otro bloque político. Este punto será tratado en extenso en la próxima sección del trabajo.

Cabe señalar que este tipo de estudio donde se cruzan datos locales (en el ámbito de lo económico y social) con resultados electorales, padece de una dolencia crónica. Básicamente el problema radica en que el "mapa" de las polis comunales no necesariamente coincide con el mapa habitacional y geográfico de estas. Esta situación tiene su origen en que un gran contingente ciudadano se inscribió para votar en el plebiscito de 1988 y desde entonces han permanecido en esta mesa de votación, independiente de si se mudaron o no a otra comuna. Si bien estas divergencias no deberían ser muy significativas en el ámbito agregado, seguramente son lo suficientemente elocuentes para agregar ruido estadístico a las inferencias llegadas. Este un problema creciente y va a seguir siéndolo más a no ser que los padrones electorales se ajusten a la realidad poblacional/ residencial del país (ver Cantillano y Navia, 2004).

\section{GÉNERO}

Si bien nunca tuvo tanta relevancia como las fracturas de clase y religión, las diferencias en las preferencias políticas entre los géneros han sido una variable crucial a la hora de explicar el grado de conservadurismo o no de un individuo. Se ha sostenido que, en términos generales, la mujer es políticamente más conservadora que el hombre (ver Norris, 2004: 116). En reiteradas ocasiones esto también fue esgrimido en el contexto chileno y de esta forma se explica por qué fue el Partido Conservador uno de los grandes abanderados en la lucha por lograr el voto de la mujer allá por los años treinta y cuarenta (ver especialmente Maza Valenzuela, 1995).

Un caso evidente ha sido la discusión interna entre los partidos de la Concertación sobre quién debería correr como candidato a alcalde en la Comuna de Santiago, quizás la más relevante simbólicamente de Chile. Los Demócratas Cristianos argumentaban que debería ser de su partido ya que tenían al Intendente, Marcelo Trivelli, mientras que los pepedistas argüían que su candidato, Jorge Schaulson Brodsky, era mucho más competente. Finalmente, el PDC accedió a retirar la nominación de Trivelli a cambio de concesiones del PPD en otras comunas. 
Sin embargo, la evidencia internacional contemporánea nos empuja a conclusiones contradictorias en cuanto a las diferencias entre los géneros a la hora de volcarse por los bloques ideológicos. Existen diferencias estadísticamente significativas en el voto de la mujer en distintos países: cuando en Israel por ejemplo la mujer tiende a apoyar en mayor medida a partidos conservadores, en otros países como Bélgica, Canadá o Estados Unidos estas tienden a votar por no-conservadores (Norris, 2004: 111).

En esta sección estudiaremos si efectivamente el voto femenino en Chile tiende a ser más de derecha que el voto de los hombres a los comienzos del siglo XXI ${ }^{10}$. Aún más, poco se ha investigado si la mujer tiene algún tipo de solidaridad femenina que la inclina a apoyar más a las mujeres que a los hombres independientemente de la postura ideológica de las candidatas. Esto iluminará un aspecto crucial a la hora de determinar diferencias o no en la conducta electoral de este contingente mayoritario del electorado.

Una de las (pocas) virtudes del sistema electoral chileno para el investigador es que las mujeres y los hombres votan en mesas separadas en todas las elecciones. En esta sección del trabajo se ha combinado el porcentaje del voto que recibió cada uno de los candidatos a concejal en cada uno de los municipios, así como el voto que recibió cada uno de los candidatos a la diputación en los distritos electorales, controlando por el porcentaje de inscritos de mujeres y hombres. De esta forma obviamos el problema de cuantas mujeres corren en cada uno de los municipios y distritos.

Para calcular si la mujer vota distintamente por los bloques políticos hemos tomado el porcentaje de votos de mujeres que recibe cada candidato en cada localidad y hemos restado ese porcentaje al porcentaje de mujeres que se encuentran registradas es esa localidad, por eso del enorme universo de análisis (más de 6500 observaciones). Si la resultante es positiva, esta indica que porcentaje más de votos recibió el candidato desde el conglomerado de las mujeres y si es negativa significa que recibió menos votos de mujeres que lo que se hubiese esperado de votar igual. Estas variaciones son significativas ya que si bien el promedio de inscritos a nivel nacional entre hombres y mujeres es cercano al $50 \%$ para cada uno de los grupos, existen diferencias significativas: Por ejemplo en el municipio de Providencia en la región Metropolitana el porcentaje de mujeres inscritas es de 59\% del total, cuando en los municipios de Río Verde o Timaukel en la doceava región este porcentaje desciende a 24\%. La Figura 2 muestra una primera imagen de las variaciones de los votos (en términos porcentuales) que recibió de las mujeres cada conglomerado político controlando por tipo de elección.

10 Esta apreciación entre conservador y de derecha puede ser confusa. El caso del PDC se podría entender que como partido confesional sea más conservador que por ejemplo Renovación Nacional. Por esta razón, en este trabajo me refiero a "derecha" para dar cuenta donde se ubica el partido respecto al gobierno vs. oposición. 
FIGURA 2: Distribución del voto femenino por bloque

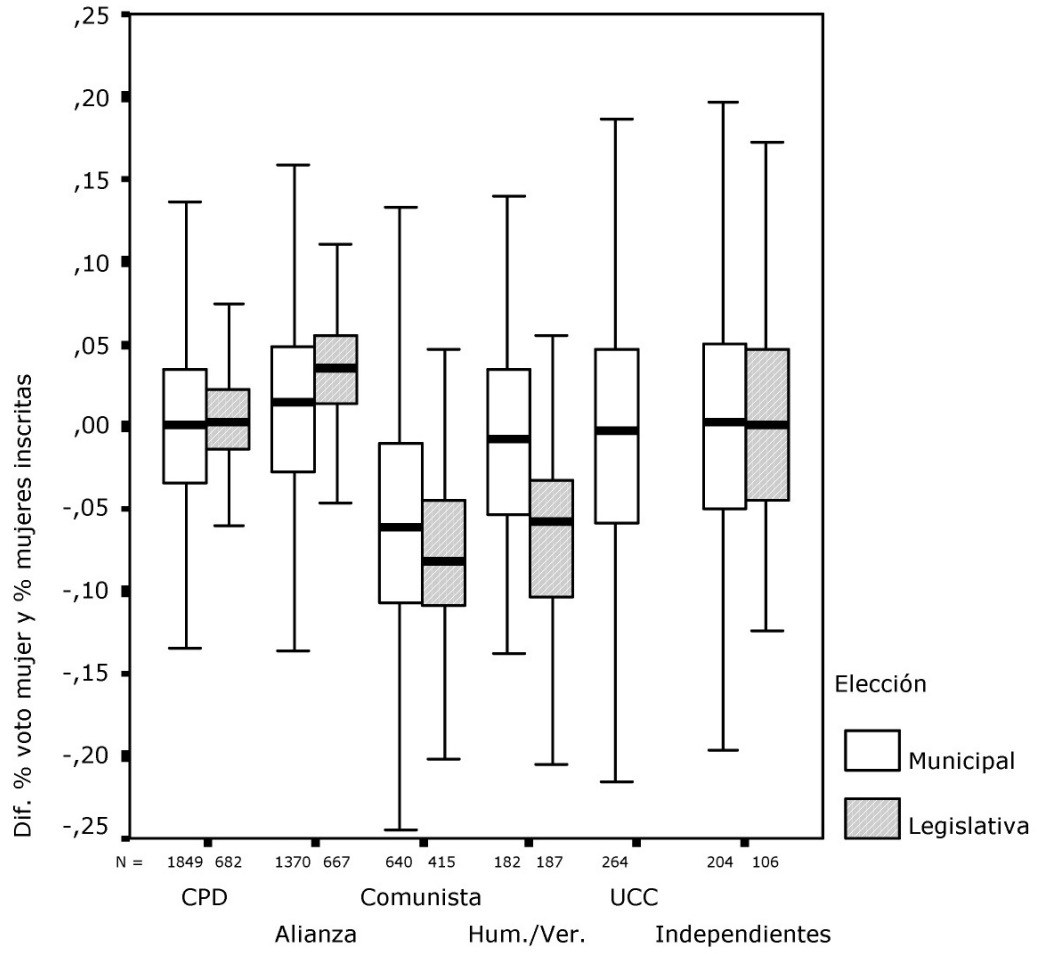

BLOQUE

En la Figura 2 podemos observar claramente que el voto de mujeres al Partido Comunista y la Coalición Humanista Verde es notoriamente más bajo que el porcentaje de los otros bloques. La Concertación de Partidos por la Democracia no presenta, aparentemente, una diferencia significativa en recibir más o menos votos del mayor contingente social del país, las mujeres. ¿Son estas tendencias estadísticamente significativas? Para responder esta pregunta testearemos con una regresión multivariada en qué medida el voto de la mujer tiende a inclinarse más por uno u otro bloque político además de controlar si la mujer vota de forma distinta regionalmente.

El Modelo 1 de la Tabla 4 tiene como variable dependiente la diferencia entre el voto recibido por cada candidato/a de las mujeres y la cantidad de mujeres inscritas en el municipio y las variables independientes son: pertenencia a los diversos bloques políticos, las regiones y tipo de elección ( $1=$ =egislativa, $0=$ municipal). El modelo 2 tiene las mismas variables dependiente e independientes, pero incluye además una variable que da cuenta del sexo del candidato en consideración ( 1 =hombre, $0=$ mujer), pudiendo así estudiar si las mujeres votan más a las mujeres que a los hombres, ceteris paribus. 
TABLA 4: Determinante del voto de mujeres

\begin{tabular}{|c|c|c|c|c|}
\hline & Modelo 1 & & Modelo 2 & \\
\hline (Constante) & -.003 & .004 & $.017^{* * *}$ & .004 \\
\hline Legislativas & .003 & .002 & $.005^{\star *}$ & .002 \\
\hline Concertación & .004 & .003 & .004 & .003 \\
\hline Alianza por Chile & $.021^{* * *}$ & .003 & $.020 * * *$ & .003 \\
\hline Comunistas & $-.059 * * *$ & .004 & $-.060 * * *$ & .004 \\
\hline Humanistas / Verdes & $-.029 * * *$ & .005 & $-.032^{* * *}$ & .005 \\
\hline I Región & $.025^{* * *}$ & .005 & $.021^{* \star *}$ & .005 \\
\hline || Región & $.013^{*}$ & .006 & $.012^{*}$ & .006 \\
\hline III Región & $-.012^{*}$ & .006 & $-.014^{*}$ & .006 \\
\hline IV Región & $.011^{* *}$ & .005 & $.011^{*}$ & .005 \\
\hline V Región & -.005 & .003 & $-.007^{*}$ & .003 \\
\hline VI Región & $-.011^{* * *}$ & .004 & $-.011^{* * *}$ & .004 \\
\hline VII Región & -.006 & .004 & -.006 & .004 \\
\hline IX Región & .004 & .004 & .004 & .004 \\
\hline X Región & -.002 & .004 & -.001 & .003 \\
\hline XI Región & $.046^{* * *}$ & .006 & $.046^{* * *}$ & .006 \\
\hline XII Región & $.054^{* * *}$ & .006 & $.052^{\star \star \star}$ & .006 \\
\hline Región Metropolitana & $-.011^{* * *}$ & .003 & $-.013^{* * *}$ & .003 \\
\hline Hombre (Hombre =1, Mujer $=0$ ) & - & - & $-.023^{* * *}$ & .002 \\
\hline $\mathrm{R}^{2}$ & .167 & & .178 & \\
\hline $\mathrm{R}^{2}$ Ajustado & .165 & & .176 & \\
\hline $\mathrm{F}$ & $77.247^{* * *}$ & & $78.414^{* * *}$ & \\
\hline $\mathrm{N}$ & 6565 & & 6539 & \\
\hline
\end{tabular}

${ }^{*} \mathrm{p}<.05 ;{ }^{* *} \mathrm{p}<.01 ;{ }^{* * *} \mathrm{p}<.005$

Los resultados de estas regresiones multivariadas satisfacen las expectativas teóricas respecto a la solidaridad de género a la hora del voto. Basándonos en el coeficiente positivo y significativo de la Alianza en el modelo 1 y tal como se ha descrito con anterioridad, la mujer en Chile tiene estadísticamente hablando una inclinación más de derecha que el hombre. Esto no significa que la Concertación sea más masculina que la Alianza. La carencia de significancia estadística en la Concertación no nos permite aventurarnos en conjeturas de esta índole. Sí, en cambio, las tendencias observadas en la Figura 2 respecto al voto de la mujer al Partido Comunista y al Partido Humanista son estadísticamente elocuentes. Más aún, en el Modelo 2 hemos incluido una variable dicotómica que representa el género del candidato/a. El coeficiente negativo y altamente significativo de esta variable nos permite evidenciar que efectivamente existe una suerte de solidaridad de género. En otras palabras, si bien la mujer tiene una leve inclinación por emitir un voto de derecha, simultáneamente este voto tiende a favorecer a sus pares de género. 


\section{DISCUSIÓN}

Este artículo ofrece una nueva oportunidad de estudiar el cambio de clivajes en el Chile postautoritario usando evidencia electoral y socioeconómica que va más allá de elecciones presidenciales y datos de opinión pública. La evidencia planteada en esta investigación nos permite determinar que las condiciones socioeconómicas, medidas a través del desarrollo humano a nivel local, tienen ciertamente efecto a la hora de determinar el porcentaje de votos recibido por cada una de las coaliciones mayoritarias en Chile (Concertación y Alianza). En términos generales, la Concertación obtiene mejores resultados en las comunas de mayor índice de desarrollo humano y la Alianza en comunas con niveles más bajos de desarrollo humano. Por cierto, esta relación lineal no es nueva en el mundo; países como Israel y Suiza presentan una relación inversa entre nivel educativo y voto por la derecha, así como entre nivel de ingresos y votos recibidos por los partidos de derecha ${ }^{11}$. En otras palabras, cuanto menor es el nivel educativo y menor el ingreso, mayor es el voto a partidos de derecha. Sin embargo, este trabajo va más allá del estudio de los efectos lineales entre estas variables y comienza a estudiar formulaciones más complejas. Si consideramos los efectos curvilíneos, la Alianza por Chile vota mejor en las comunas con valores extremos de IDH y la Concertación dentro de las comunas con valores medios. En otras palabras, la Concertación presenta una distribución con forma de $U$ inveritda $(\cap)$ y la Alianza lo opuesto $(\cup)$.

Respecto al voto del grupo mayoritario del electorado chileno, las mujeres, se puede afirmar que definitivamente las mujeres tienden a votar más a las candidatas, incluso controlando por pertenencias a los distintos bloques políticos. En otras palabras, la mujer tiende a votar más a la mujer candidata por más que esta pertenezca a un bloque político distinto. No creo que sea muy aventurado suponer que, en vísperas de las elecciones presidenciales del 2005, con presumibles candidatas mujeres a la presidencia por parte de la Concertación, más de un lector se pregunte si el efecto solidaridad de género es más relevante que la inclinación de las mujeres por la derecha. Si bien en términos estadísticos se podría conjeturar el diferencial en las magnitudes de los efectos, no creo conveniente augurar qué pasará, por la simple razón de que en la elección presidencial se juega mucho más que el mero efecto de género.

Ciertamente muchos problemas metodológicos se le presentan al investigador a la hora de hacer este tipo de estudios. Este trabajo no pretende tener, obviamente, la última palabra sobre la conducta electoral de la ciudadanía chilena en estas dos elecciones, sino que pretende comenzar a esbozar teorías basadas en evidencia empírica de resultados electorales.

\section{REFERENCIAS}

Agüero, Felipe, Eugenio Tironi, Eduardo Valenzuela, y Guillermo Sunkel. 1998. "Votantes, Partidos e Información Política: La Frágil Intermediación Política En El Chile Post-Autoritario". Revista de Ciencia Política XIX (2): 159-193.

Aldunate, Adolfo. 1981. "Antecedentes Socioeconómicos y Resultados Electorales". En Estudio sobre el Sistema de Partidos en Chile. Santiago de Chile: FLACSO.

Almond, Gabriel y Sidney Verba. 1963. The Civic Culture. Princeton: Princeton University Press.

11 Obviamente, aunque muy alejado del foco de este trabajo, sería muy interesante explorar el por qué estos países tienen estos patrones de conducta electoral. 
Altman, David. 2002. "Popular Initiatives in Uruguay: Confidence Votes on Government or Political Loyalties?" Electoral Studies 21 (4): 617-630.

Angell, Alan. 2003. "Party Change in Chile in Comparative Perspective". Revista de Ciencia Política XXIII (2): 88-108.

Araos, María Raquel y Eduardo Engel. 1989. “Desempleo, Votación Histórica y el Plebiscito de 1988”. Estudios CIEPLAN 27 (Diciembre): 5-17.

Barozet, Emmanuelle. 2003. "Movilización de recursos y redes sociales en los neopopulismos: hipótesis de trabajo para el caso chileno". Revista de Ciencia Política XXIII (1): 39-54.

Blair, Harry. 2000. "Participation and Accountability at the Periphery: Democratic Local Governance in Six Countries". World Development 28 (1): 21-39.

Blais, André y Agnieszka Dobrzynska. 1998. "Turnout in electoral democracies”. European Journal of Political Research 33 (2): 239-262.

Bowler, Shaun y Todd Donovan. 1998. Demanding Choices: Opinion, Voting, and Direct Democracy. Ann Arbor: The University of Michigan Press.

Cantillano, Priscilla y Patricio Navia. 2004. "Representación y Tamaño de los Distritos Electorales en Chile, 1988-2002". Mimeo: Universidad Diego Portales.

Carey, John. 1997. "Chile: Latin American Proportionality or Majoritarianism?" En The International Idea Handbook of Electoral System Design, editado por A. Reynolds y B. Reilly. Stockholm: IDEA.

Carey, John. 2002. "Parties, Coalitions, and the Chilean Congress in the 1990s". En Legislative Politics in Latin America, editado por S. Morgenstern y B. Nacif. Cambridge: Cambridge University Press, Pp. 222-253.

Carey, John M. y Peter Siavelis. 2003. "El "seguro" para los subcampeones electorales y la sobrevivencia de la Concertación”. Estudios Públicos 90 (Otoño): 5-27.

Coppedge, Michael. 1998. "The Evolution of Latin American Party Systems". En Politics, Society, and Democracy: Latin America, editado por S. Mainwaring, y Arturo Valenzuela. Boulder: Westview Press, Pp. 171-206.

Crewe, Ivor. 1982. "Electoral Participation". En Democracy at the Polls, editado por D. Butler, H. R. Penniman, y A. Ranney: American Enterprise Institute, Pp. 216-63.

Cruz-Coke, Ricardo. 1958. Geografía Electoral de Chile. Santiago de Chile: Editorial del Pacífico S.A.

Dix, Robert H. 1989. "Cleavage Structures and Party Systems in Latin America”. Comparative Politics 22 (1): 23-27.

Dow, Jay K. 1998. "A Spatial Analysis of Candidate Competition in Dual Member Districts: The 1989 Chilean Senatorial Elections". Public Choice 97 (3): 451-474.

Dow, Jay K. 1998. "A Spatial Analysis of the 1989 Chilean Presidential Election”. Electoral Studies 17 (1): 61-76.

Eulau, H. y M.S. Lewis-Beck. 1985. Economic Conditions and Electoral Outcome. New York: Agathon.

Fornos, Carolina A., Timothy J. Power, y James C. Garand. 2004. "Explaining Voter Turnout in Latin America, 1980 to 2000 ". Comparative Political Studies 37 (8): 1-32.

Garman, Christopher, Stephan Haggard, y Eliza Willis. 2001. "Fiscal Decentralization: A Political Theory with Latin American Cases". World Politics 53 (2): 205-236.

Gimpel, James G., Irwin L. Morris, y David R. Armstrong. 2004. "Turnout and the Local Age Distribution: Examining Political Participation across Space and Time". Political Geography 23 (1): 71-95.

Godoy, Oscar. 2003. "Parlamento, presidencialismo y democracia protegida". Revista de Ciencia Política XXIII (2): 7-42.

González Leiva, José Ignacio. 1999. "Geografía Electoral de Chile: Comportamiento del Electorado Chilenos entre 1932 y 1992". Estudios Geográficos LX (234): 121-138.

Hibbs, D. A. Jr. 1979. "The Mass Public and the Macroeconomic Performance: The Dynamics of Public Opinion towards Unemployment and Inflation". American Journal of Political Science 23 (4): 705-731.

Jackman, Robert W. 1987. "Political Institutions and Voter Turnout in the Industrial Democracies". American Political Science Review 81 (2): 405-423.

Jackman, Robert W. y Ross Miller. 1995. "Voter Turnout in the Industrialized Democracies during the 1980s". Comparative Political Studies 27 (4): 467-492. 
Laakso, Markku y Rein Taagepera. 1979. "Effective Number of Parties: A Measure with Application to West Europe". Comparative Political Studies 12 (1): 3-27.

Lijphart, Arend. 1997. "Unequal Participation: Democracy's Unresolved Dilemma”. American Political Science Review 91 (1): $1-14$.

Luna, Juan Pablo. 2004. "Shaky foundations? The transformation of party-society linkages in post-transitional Chile and its implications for the quality of representation". Presentado en MPSA Meeting, April 15-18, Palmer House Hilton, Chicago, IL.

Mainwaring, Scott y Aníbal Pérez-Liñán. 2003. “Level of Development and Democracy: Latin American Exceptionalism, 19451996". Comparative Political Studies 36 (9): 1031-1067.

Mainwaring, Scott y Tim Scully. 1995. Building Democratic Institutions. Stanford: Stanford University Press.

Mainwaring, Scott y Mariano Torcal. 2003. "The Political Recrafting of Social Bases of Party Competition: Chile, 1973-95". British Journal of Political Science 33 (1): 55-84.

Maza Valenzuela, Erika. 1995. "Catolicismo, anticlericalismo y extensión del sufragio a la mujer en Chile". Estudios Públicos 58 (Otoño): 137-197.

Nannestad, Peter y Martin Paldman. 1994. "The VP-function: A survey of the literature on vote and popularity functions after 25 years". Public Choice 79 (3-4): 213-245.

Navia, Patricio. 2002. "Mayoría electoral versus triunfo electoral". En Chile 2001-2002: Impactos y desafíos de las crisis internacionales, editado por FLACSO. Santiago: FLACSO-Chile, Pp. 127-147

Navia, Patricio. 2003. "Comportamiento electoral comunal 1996-2001. Con miras a las municipales del 2004". Perspectivas 6 (2): 267-291.

Navia, Patricio. 2004. "Participación electoral en Chile 1988-2001". Revista de Ciencia Política 24 (1): 81-103.

Nolte, Detlef. 2003. "El Congreso chileno y su aporte a la consolidación democrática en perspectiva comparada". Revista de Ciencia Política XXIII (2): 43-68.

Norris, Pippa. 2004. Electoral Engineering: Voting Rules and Political Behavior. Cambridge: Cambridge University Press.

Ortega, Eugenio. 2003. "Los partidos políticos chilenos: Cambio y estabilidad en el comportamiento electoral 1990 - 2000". Revista de Ciencia Política XXIII (2): 109-147.

Panzer, John y Ricardo Paredes. 1991. "The Role of Economic Issues in Elections: The Case of the 1988 Chilean Presidential Referendum". Public Choice 71 (1-2): 51-59.

PNUD Chile. 2000. Desarrollo Humano en las Comunas de Chile. Santiago: PNUD.

Powell, G. Bingham. 1986. "American Voter Turnout in Comparative Perspective”. American Political Science Review 80 (1): 17-43.

Radcliff, Benjamin. 1992. "The Welfare State, Turnout, and the Economy: A Comparative Analysis". American Political Science Review 86 (2): 444-454.

Rahat, Gideon y Mario Sznajder. 1998. "Electoral Engineering in Chile: The Electoral System and Limited Democracy". Electoral Studies 17 (4): 429-442.

Rallings, C., M. Thrasher, y G. Borisyuk. 2003. "Seasonal factors, voter fatigue and the costs of voting”. Electoral Studies 22 (1): 65-79.

Rius, Andrés. 1992. "El gobierno, la economía y el hombre de la calle". SUMA 7 (13): 7-35.

Rowland, Allison M. 2001. "Population as a Determinant of Local Outcomes under Decentralization: Illustrations from Small Municipalities in Bolivia and Mexico". World Development 29 (8): 1373-1389.

Schneider, Friedrich y Jorg Naumann. 1982. "Interest groups in democracies - How influential are they? An empirical examination for Switzerland". Public Choice (38): 281-303.

Siavelis, Peter. 1997. "Continuity and Change in the Chilean Party System". Comparative Political Studies 30 (6): 651-674.

Siavelis, Peter. 2000. The President and Congress in Post-Authoritarian Chile: Institutional Constraints to Democratic Consolidation. University Park: The Pennsylvaria University Press.

Siavelis, Peter. 2002. "Exaggerated Presidentialism and Moderate Presidents: Executive-Legislative Relations in Chile". En Legislative Politics in Latin America, editado por S. Morgenstern y B. Nacif. Cambridge: Cambridge University Press, Pp. 79-113.

Siavelis, Peter. 2004. "Sistema electoral, desintegración de coaliciones y democracia en Chile: ¿El fin de la Concertación?" Revista de Ciencia Política XXIV (1): 58-80. 
Sundar, Nandini. 2001. "Is Devolution Democratization?" World Development 29 (12): 2007-2023.

Tironi, Eugenio y Felipe Agüero. 1999. “Sobrevivirá el nuevo paisaje político chileno?” Estudios Públicos 74 (Otoño): 151168.

Valenzuela, Arturo. 1994. "Party Politics and the Crisis of Presidentialism in Chile: A Proposal for a Parliamentary Form of Government". En The Failure of Presidential Democracy: The Case of Latin America, editado por J. Linz y A. Valenzuela. Baltimore: The Johns Hopkins University Press.

Valenzuela, Samuel J. 1995. "Orígenes y transformaciones del sistema de partidos en Chile”. Estudios Públicos 58 (0toño): 577.

Valenzuela, Samuel J. 1999. "Reflexiones sobre el presente y futuro del paisaje político chileno a la luz de su pasado: Respuesta a Eugenio Tironi y Felipe Agüero". Estudios Públicos 75 (Invierno): 275-290.

Wolfinger, Raymond y Steven Rosenstone. 1980. Who Votes? New Haven: Yale University Press.

David Altman (Ph.D. Universidad de Notre Dame, 2001) es actualmente profesor auxiliar del Instituto de Ciencia Política de la Pontificia Universidad Católica de Chile. Sus trabajos han aparecido en Electoral Studies, Party Politics, Democratization, International Review of Public Administration, Revista Uruguaya de Ciencia Política y Cuadernos del CLAEH entre otros.

(E-Mail: daltman@uc.cl) 